\title{
Critique of Computational Reason in the Natural Sciences*
}

\author{
Giuseppe Longo \\ Département d'Informatique \\ CNRS - Ecole Normale Supérieure et CREA, Polytechnique, Paris \\ http://www.di.ens.fr/users/longo
}

September 8, 2009

\begin{abstract}
In this text, we will attempt to shortly highlight certain constitutive principles of the particular form of knowledge provided by the digital machine, the modern computer, in its relationship to Mathematics (from which it originates) and to the Natural Sciences (Physics and Biology). Our basic thesis is that the historical and conceptual richness of the theory which enabled the concrete realization of this extraordinary type of machine is far from being neutral or transparent with regard to reality. Specifically, we will see that the digital machine proposes causal structures and the breakings of symmetry which generate them as being the central structures of the intelligibility of nature. This will enable to point out a distinction between "imitation and "modeling in terms of simulation or formalization, and therefore enable to highlight the limits and the potentialities of digital simulation.
\end{abstract}

\section{From the alphabet to the machine}

The extraordinary innovation to which we are confronted today is a machine which is the result of a very specific historical evolution. This machine did not exist "before", in the way in which there were no mammals on earth 300 million years ago. It is within the evolutive system's dynamic, which constantly produces novelty, that mammals emerge: nothing miraculous, only a very complex mixture between invariance and variability, continuity and change, which are in part random, and in part not yet properly classifiable into current physical categories of determination. In a similar or more complex fashion, human history develops, and within it, with a continuity/ discontinuity which is rich in terms of common practices, of language, and of symbolic culture, we invented this machine, which is in the process of changing the world. Such a machine is the culminating point of a very specific process which begins with language, but

*In "Fundamental Concepts in Computer Science" (E. Gelenbe and J.-P. Kahane, eds.), Imperial College Press/World Scientific, 2009, Text originally written in Italian as Lezione Galileana, Pisa, 25 ottobre 2006. A French version is also downloadable: http://www.di.ens.fr/users/longo 
which is mainly influenced by the birth and development of the alphabet: the digital machine is at first an alphabetic machine, and then a logical and formal one. In short, it is an invention which is both extraordinary and contingent to our culture, which is marked by the birth of the alphabet, of Cartesian rationality, of Fregean logic, of Hilbertian formalism.

So lets enunciate the problem of considering what is the impact of such a machine on the construction of knowledge. The machine is indeed not neutral; it imposes upon one who uses it a history and a logic, an organizing view of phenomena. The most deleterious cultural attitudes are of those who remain naive before the novelty brought on by evolution and history (or that we bring into it): not knowing how to live according to our own knowledge, not knowing how to appreciate the originality of our own knowledge, and projecting our latest invention onto the past, as if, while rich in human history, it was already in the world, or if it were an accurate image of it. And continuing to say: the universe is a big computer, or... each physical or biological process is a computation. Or that Turings theory is "complete" and "maximal": even a cells activity or quantum computing can be reduced to it. This is a pretense to having the "Definitive theory", in an Aristotelian sense.

And, most of all, we do not consider the originality of this extraordinary science and of this technology which, by organizing our view upon phenomena in their own way (and in their own image), help and guide us in the acquisition of knowledge. The machine, as other instruments in the past did and even more so, deeply impacts our relationship to science, as the alphabet and the printing press have transformed and impacted our societies, even the way in which we construct knowledge. I will not dwell on all the themes we have evoked, and I will only point out the view which computer science proposes, one imbued by a very effective organization of knowledge into little boxes, into bits, into pixels, into a discrete or sometimes absolute exactitude, with no smoothness, no fuzziness, no gestalt, no alea. Or with at best some very important imitations (in a sense to be specified below) of such components of the world and of knowledge, but ones which are forced or biased by their own logic.

So I would like to readdress the fact that the roots of this machine are very old and can be found in the alphabet. First of all, 5000-6000 years ago, the alphabet was, for different reasons, an invention comparable to the computermediated discretization of knowledge we have now performed. Think of the originality of these first social groups from Mesopotamia who fractioned the linguistic flux, a continuous spoken song, marking certain pitches as first consonants [Herrenschmidt C. et al. 1996]. It was the onset of a development and of a culture which were quite different to those inherent to the hieroglyphic writing of ideograms which proposed concepts or evoked whole images, situations, or feelings, by means of drawings. Conversely, the alphabet discretizes, subdivides continuous language into insignificant atoms, into the bits which are letters. This constitutes an extraordinary leap of abstraction by man, a way of representing linguistic interaction which absolutely did not exist before and which will mark human culture by the (re-)construction of meaning from elementary and simple signs without meaning, signs that were highly abstract as such. Moreover, and this is crucial, meaning is reconstructed through sound: the alphabet is phonetic. Meaning is provided by the reproduction of sound, and not by the evocation of an image or of a concept, a huge revolution. In computer science 
terms, the phoneme is the alphabets compiler, or the "interpreter" if you wish, and it produces meaning. By means of the drawing, hieroglyph or ideogram, the evocation of a concept, of an emotion or of a god is conveyed in silence. The road sign, an ideogram, imposes a direction, an order, or a prohibition in the visual immediateness of a significant evocation: it is understood, acted upon, without the production of sound, not even mental. If on the other hand, for example, the indication which prohibits turning right by means of an evocative sign is written, as is often the case in the US, you will necessarily pronounce the words "no right turn", at least in thought. Producing a phoneme, one which is exclusively mental when reading in silence, is necessary to obtain meaning, and we all go through the difficulties posed by our first attempts at decryption during childhood, which is necessary performed vocally when learning (it would appear that silent reading was not invented until the III ${ }^{\text {rd }}$ or IV th century: before that, western man would always read aloud). Musical writing will undergo the same process and the expert musician mentally hears music when reading it, even silently, just as we hear alphabet-based significant words, because they resound.

\section{The elementary and the complex}

With the observations on the alphabet's role, I took up the detailed and profound observations made by Herrenschmidt in Paris, by Sini and his school in Milan, as well as to other authors: alphabetic fractioning will orient human culture in a very strong way. Lets see how and why this has anything to do with computer science.

The alphabet is extraordinarily effective: it forces into shape, it canalizes and organizes thought, it structures knowledge. Firstly, it introduces an original form of dualism: here, notation, there, signification, linked by means of the phoneme, but also independent (with the ideogram, signification is immanent to the drawing). Then, there is the conception according to which, in order to understand the world, it is necessary to fraction it into elementary and simple components. Democritus designated atoms by means of the letters of the alphabet: the universe is constructed in the image of our invention, the alphabet, and is formed by the combination of elementary and simple components, which are indivisible, like letters. Today, the genome is still described by means of letters of the alphabet. Atoms, or genomes bases and molecules, aggregate between each other, and then, there emerges, as a pop-out, the physical object, the phenotype, the behavior: just how meaning emerges by aggregation of letters and by means of the phoneme. And man projects, once more, this manner of reconstructing and of talking about the world, onto the absolute: he says that God (or evolution) invented the world and life in the way he constructs meaning himself with alphabetic reading, by juxtaposing signs with no signification. Once more, the alphabet is very effective and extraordinary, but it is not a neutral instrument, it imposes by its own force the paradigms which will be at the origin of western science and which are still revisited today in contemporary science. Particularly, it proposes the paradigm that Descartes, more than anyone else, placed at the center of knowledge: the elementary components of the construction of knowledge must be very simple, insecable links of the rational chain of 
Cartesian reasoning. Letters, in themselves, are indecomposable (elementary), are very simple, and do not have meaning, but when arranged together, they produce meaning that can be very complex. Such is Democrituss approach to science but also, I insist, that of Aristotle and of Descartes: intelligibility is produced by the decomposition of the universe into atoms and the discourse on the universe into simple and elementary links. It is the maximal, atomic decomposition of elements which makes the universe intelligible and discourse rigorous. This is how Galileo and Newton work and all of modern science, with an incredible effectiveness, constructs knowledge, from the elementary and the simple. It has been more productive than any other science (the Chinese come to mind, for instance) especially for making machines, though not exclusively. Clocks are made like this: they are composed and highly complex objects made from simple gears and belts, in the way XVIII ${ }^{\text {th }}$ century clockmakers would make them. And so is made the computer: the logical gates and elementary components are very simple; programming languages are composed of elementary and simple linguistic atoms, and used to make systems and programs of an extraordinary degree of complexity.

However, we are faced today with an enormous difficulty, a new challenge in terms of knowledge: in the two most innovative fields, at the difficult frontier of knowledge, Quantum Physics and Biology, the elementary is in fact very complex, and this is the great challenge to our understanding, with our being so alphabetized. We can refer to the case of strings or to the phenomena of non-separability and non-locality specific to Quantum Physics which are of an extreme level of complexity and which concern elementary components of matter. So, our projection of the alphabet upon the world, the letter-atoms of Democritus, suddenly faces an obstacle, which is for the moment insurmountable (we cannot understand Microphysics in classical or relativistic terms). The same thing is happening with the analysis of living phenomena: the cell, as elementary component of living matter (if we split it, it dies; it is no longer living) is very complex, and must be considered in its unity. Some biologists (Gould, among many others) assert that a eukaryotic cell is as complex as an elephant. Indeed, within a cell reside the same proteinic cascades, the same type of energy production (mitochondria, metabolism ...), a structuring into organs that is analogous to that which exists in a metazoan. An aspect of complexity, the objective one, is therefore similar in the elephant and in the eukaryotic cell. An animal is obviously more complex than a cell from the phenotypical point of view, but that is another type of complexity (morphological). The new challenge, the complexity of the elementary is a conceptual obstacle to our alphabetic and digital decomposition of the world, which is otherwise very effective: we have difficulties overcoming it. As in Quantum Physics, where there lacks unity with the classical or relativistic "field", we have trouble unifying the "field" of living phenomena (which we have also not yet defined) with current biochemical theories, the theories which use macromolecules and bases as words and alphabet. A reflection in this regard, thanks to the contribution (by duality) of digital computer science, could possibly help. 


\section{Imitations and models}

Let's return to the digital. So it is the strength of alphabetic culture which has given us this machine, the digital computer, as its ultimate expression, the culminating point of human, alphabetic and Cartesian invention. The machine is alphabetical, above all because everything is composed of $0 \mathrm{~s}$ and $1 \mathrm{~s}$. The basic alphabet is very simple and also has very simple elements, and it then becomes very complex, by composition. It is Cartesian because it is the maximal locus of Cartesian dualism, realized from Turing's idea in 1936: the electrical calculating machines of that era and which continued to be used until the $50 \mathrm{~s}$ did not have software that was distinguishable from the hardware. They would have multiplication implemented within them, in a way, and it would remain inscribed in the gears: the rules, one by one, would shape the hardware which was constructed ad hoc. These machines were constructed as were clocks 200 years earlier, only being more complex. Turing's idea, having some predecessors, was to clearly and mathematically distinguish, in the abstract machine, the hardware, as multi-functional physical material, from the software. Then the theory of programming, completely independent from hardware, emerged from specific electronics. The main idea making Computer Science possible is the portability of software, in its independence from hardware: a program is written, is moved from one machine to another, and it works. It can be sold independently. There exists a line of work which I have practiced for a long time, that of mathematical logician in Programming Theory, and which is completely independent from the analysis of hardware. Naturally, to a monist like myself, this has nothing to do with the world, and even less to do with living phenomena: it is rather the modern image of mind/body Cartesian dualism, with its lot of metempsychosis (the transferal of programs and operating system from a dying computer to another) which enjoys a great success in Artificial Intelligence and in bad Sci-Fi movies. I do insist however that such a paradigm is rich in knowledge, beginning with the construction of the alphabet, maybe the first truly dualist experience of man, as we were saying: insignificant sign and signification, each being highly distinguished from the other. And I would recall Aristotle once more. He outlines a theory of memory and of reasoning based on the alphabet according to which, he asserts, the unfolding of reasoning is like the marking, the stamping of "alphabetic signs on the body, as on a wax tablet" [Cappuccio M. 2003]: it is the alphabetic signs that enable reasoning with their purely formal dynamic which is independent from meaning. Thought resides in the mobile impression of signs. This constitutes Aristotle's and Turing's alphabetic model of reasoning (the Turing machine could be called the Aristotle-Turing machine): letters which move and which are impressed upon matter (living matter, as on wax), or on the ribbon of a Turing machine, the prototype of the modern computer. From then on, we get to a machine which represents everything, through Cartesian and atomist reasoning, by means of a sequence of letters without signification. This way of understanding human (and animal) intelligence canalizes the view on reality with great effectiveness, but it is biased, by a bias resulting on the one hand from its dualistic aspects (that I would qualify as ferocious) and on the other hand, from the fact of only proposing intelligibility by means of the reduction to the simple and elementary, the sequence of ultimate and very simple signs/atoms, without meaning. Once more, this paradigm was very rich for (classical) physico-mathematical 
knowledge and technologies, particularly, but today it remains confronted to the obstacle of this very complex, non alphabetical elementarity which we find in Quantum Physics and Biology, and which is rich in entanglements and causal circularities specific to these two theoretical frames.

The first consequence to draw from these considerations is an invitation to a lot of circumspection when using the computer as instrument of intelligibility. In other words, it is important to not do like some colleagues, in the Natural Sciences too, who consider as valid everything they see on the screen, the models which the machine enable. To this day, the richness of the digital simulation is such that it deserves a fine, an epistemological analysis in particular, precisely in order to do better and more.

I would like to note that Turing himself, in this regard, introduced an implicit but fine distinction between "imitation" and "model", with the intuition, after 1948, of an intrinsic limit to his machine which he will qualify as "Laplacian" in the 50s. To understand what he meant, let's take an example, the double pendulum. It consists in a physical object which is very sensitive to the initial conditions. It may be formalized by two very informative differential equations determining its movements: two rods connected by a pivot, two weights... from the mathematical viewpoint, there are only two variables, one single law, gravitation, and despite that... chaos. From the intelligibility standpoint, those who know non linear systems will immediately understand that this artifact is very sensitive to initial conditions (the Lyapounov coefficients can tell the mathematician this). If we launch the pendulum from certain initial values, inevitably, within the range of possible physical measurement, and if we then relaunch it, within the same range, that of observability, then a variation, a fluctuation below the observable (or non-measurable, for example thermal fluctuation) suffices to give the double pendulum a completely different course. The double pendulum, a perfectly deterministic machine (it is only determined by two equations!), is sensitive to minor variations, below the threshold of observability: it is a typical chaotic deterministic system, as there are many of them ${ }^{1}$.

\footnotetext{
${ }^{1}$ The notion of deterministic chaos is mathematically very solid and is 110 years old (Poincaré), even if its modern definitions date back only to the 60s and 70s. These definitions may be summarized as follows: a deterministic physical system (a system considered to be determined or determinable by an evolution function or a finite number of equations, such as a double pendulum, the planetary system, a coin tossed within a gravitational field over a mathematically describable area...) is chaotic when it is "topologically transitive" (there are dense orbits, that is, orbits that go everywhere whitin the border conditions), when it has a "dense set of periodic points" and is "sensitive to the initial conditions". These properties can be described with mathematical rigor (let's note that such is the case for the three abovementioned systems: so is the solar system, according to recent results, see [Laskar J. 1994]. In what concerns images, the attractor of a non-linear system, even in one dimension (an equation, such as $\left.x_{n+1}=4 x_{n}\left(1-x_{n}\right)\right)$, truly evokes what Plato would also qualify as chaos (superimposition of lines or points, "creazy" oscillations...). Deterministic chaos is therefore not an oxymoron, despite of some bad vulgarization, but a very solid mathematical notion. As a great instrument of intelligibility, it enables to understand classical randomness, as opposed to quantum randomness, see [Bailly F., Longo G. 2007], as a determination which does not imply predictability (nor iterability: a classical process is random when, in general, it does not follow the same "trajectory" despite being iterated with the same initial conditions, within the limit of physical measure); this is the great shift relatively to Laplace's conjecture, according to which "determination implies predictability".
} 
On the other hand, when observing digital simulations (an implementation can be found on http://www.mathstat.dal.ca/ selinger/lagrange1/doublependulum.html), we can clearly see dense trajectories: thanks to the simulation, we can make the pendulum oscillate long enough and can observe that it tends to cover all the space of possible trajectories. This is an aspect of chaos. Yet, when we click on "restart" (relaunching the pendulum with the same initial data), it again takes the exact same trajectory. With a real and physical pendulum however, this is absolutely impossible. If we have a good pendulum at hand, one sufficiently insensitive to friction, but a physical pendulum, not a virtual one, then even the thermal fluctuation, which is inherent to the physical process, suffices to launch it upon another trajectory, if reinitialized. Therefore, this excellent imitation which tells us so many very useful things, what does it actually propose? On the one hand, it shows us the density of the trajectories, typical of deterministic chaos, but on the other hand, it causes us to loose an essential piece of information: in a dynamic (non-linear) system, it especially occurs that, once reinitialized, the systems never take the same "trajectory". And this because of "principles" which are inherent to Physics (modern Physics): physical measurement is always an interval and the (inevitable) variation, below the threshold of measurement, suffices to very quickly produce a different evolution. The analysis of the equations within the continuum leads to an understanding of this random aspect of chaos, whereas computational imitation makes it disappear completely, by the discrete nature of its data types. Only tricks and stratagems (pseudo-synchronization with distant watches, pseudo-random generators introduced ad hoc) can imitate, but not modelize, the physical phenomenon. That is, they can deceive the observer of virtual reality, as Turing hopes to deceive the observer in the man/machine/woman imitation game, as he called it, but they can not propose a physico-mathematical "model" of the possible causal structure of the physical phenomenon, as I would like to explain. For those with a phsyico-mathematical sensitivity, it is almost funny to see a computer simulation in which, by giving the same numeric initial values, a double pendulum, or a turbulence, will take the exact same trajectory, because this makes no physical sense. This is a case of imitation, as Turing would rightfully say. Indeed: this term which I have used in a few articles (downloadable from my Web page), has been suggested by Turing who, after 1948, began to take interest in dynamical systems, and stopped asserting that his machine was a huge brain. In 1950, he wrote an article on how to imitate human behavior using his machine (the imitation game between the machine and. . . a woman: can they be distinguished in a teletype-mediated dialog? Turing had a complex relationship with women and was homosexual). In 1952, though, he published an article on morphogenesis which proposed a very original non-linear system of actionreaction and dynamic diffusion, in which he provided what he called a model of the physical phenomenon in question. He thus sought to propose a structure of determination, by means of the equations describing causal interaction in the action-reaction process.

I hope that the implicit distinction to be found in Turing and which I have developed here can be useful to better understand what is done thanks to the digital machine: so I will return to it.

A model (a physico-mathematical one) is an attempt to express a possible 
structure of physical causality. For example, Newton considers movement (of planets and of masses) and writes equations, among which $f=m a$, that make the dynamics intelligible. That is, he makes a formidable proposition, as follows: force causes acceleration, with mass as proportionality coefficient. He proposes, with his equations, a structure of causality which will enable to deduct, among other things, the Kepler orbits. From there on, extremely interesting and fertile relationships have been developed between Physics and Mathematics. We have learned to make organizing propositions of the physical world like never before. Someone who, like Newton, is mainly preoccupied with Metaphysics, believes that the latter is reality as such; someone more laic would rather say: this is construction of knowledge, with all the objectivity of modern science, but with its own specific conceptual and practical instruments, and so with its own dynamic and evolution. In Einstein's relativity, this causal relationship is profoundly altered and, in a certain sense, it is inversed: it is the acceleration over a geodesic in curve Riemannian varieties which, by producing a field, induces a force. A formal symmetry, the equation, is broken in various ways (reversed in some cases), thus changing intelligibility (and Physics). These are great successes of the relationship between Physics and Mathematics. In reference to the mathematical modelization of physical phenomena, in [Bailly F., Longo G. 2006], we further highlight the role of symmetries and their breakings in the analysis of physical causality.

Imitation is something different, and Turing puts it quite nicely: imitation is a construction which does not claim to make the phenomenon intelligible, by proposing a causal structure for it (or better, symmetries or symmetry breakings). Imitation resembles causality, it can even be indistinguishable from it, but it does not assume any obligation towards it, towards this aspect of physical intelligibility of what is observed or imitated. For example, if you throw a coin, you will get a sequence of $0 \mathrm{~s}$ and $1 \mathrm{~s}$ : you will then be able to imitate the process, the sequence, with a random number generator, on a computer. You will have an imitation in the sense where the distribution of probabilities of $0 \mathrm{~s}$ and $1 \mathrm{~s}$ is analogous and indistinguishable, for a sequence of reasonable length. One can say that this imitation is excellent, but it has nothing to do with the modelization of a toss of a coin. Because one the toss of a coin - is the process related to a deterministic system, which is extremely sensitive to the conditions of the environment, to the slightest variation in the parameters at play, and is therefore another paradigmatic example, although a bit different from the previous, of deterministic chaos, and a paradigm of randomness by the extreme sensitivity to border conditions. The other, the computers pseudo-generator of random numbers, is also a system that is deterministic, but not chaotic: a computer's random number generator is a short one line program which multiplies sines and cosines, rounds up the results, in manner inherent to the machine, and produces a sequence of $0 \mathrm{~s}$ and $1 \mathrm{~s}$, seemingly distributed at random. But there is nothing random to the process, as opposed to the completely unpredictable toss of a coin: if you click "restart" while leaving all of the machine's parameters identical (something quite possible, even most easy), the supposedly random series will in fact be an identical reproduction, according to the arithmetic law/determination written into the program, which unfolds in the realm of the discrete. The process is Laplacian and is predictable, by iteration, identically, as a time symmetry shift. You will never manage to reproduce such 
a succession of 0s and 1s with a second launch sequence of the same coin. The causal structures differ profoundly, even if the imitation is excellent.

The essential difference resides in the fact that the basis of digital data is exact; it has, "naturally", the discrete topology, that is, its access proceeds bit by well separated bit. Physical measurement is, conversely, and by physical principle, always an interval that is well represented by continuous Mathematics (where discrete topology is not "natural"). In chaotic deterministic systems, a fluctuation/variation below the interval of measurement induces radically different evolutions for the system. This, Turing observes in the $50 \mathrm{~s}$, is theoretically avoidable in the Discrete State Machine he has invented and as he named it in those years (and such is also the case in practice: iteration and portability of software, a fundamental form of iteration, work; see [Longo G. 2007] for references and further reflections; note that in the ' 30 's Turing had called his machine the Logical Computing Machine).

\subsection{Models, processes and unpredictability}

The notion of chaotic dynamics is a mathematical notion and, as we recalled above, it is possible to give a precise definition of a dynamical chaotic system, determined by one or several equations or, more directly, by an evolution function (an endomorphism of a metric or topological space) with the properties enumerated in the note. Unpredictability, on the other hand, is given in the interface between a physical process and Mathematics: in order to be able to speak of unpredictability, it is necessary to try to predict (pre-dicere, in latin), by means of Mathematics, the evolution of a phenomenon, a physical one, typically. A process is not unpredictable in itself, without an attempt to account for it or to predict it by means of a mathematical system. On the other hand, a system of functions or a mathematical function is not unpredictable in itself either, as some claim. To the contrary, theorems demonstrate that each Cauchy problem, a very large class of differential equations or, more generally, any reasonable system of equations (or of functions) that is expressible and has a solution, has a computable one. And it is indeed necessary to look attentively to find a system of equations with computable coefficients, of course, having non-computable solutions (see [Hoyrup M., Kolcak A., Longo G. 2007] for references). In fact, staying in the field of Mathematics, we compute, and if we have good theorems of existence (and unicity, if possible) of solutions, we can predict the evolutions, point by point, each time that computable data is provided to the given system. Mathematics is written in a finite and effective language, even when speaking of infinity: it is very difficult, if we do not make efforts by using stratagems and tricks, to mathematically provide a non-computable function or number (we only know of diagonal tricks, since Turing).

In general, thus, any mathematical determination (by a system of equations, by an evolution function) is computable, hence predictable, when it is given a computable input. And randomness, within Mathematics, only happens at infinity. In short, an infinite sequence is Martin-Löf random when it passes all the effective tests (for a recent survey, see [Rojas C. 2007]): its initial (finite) segments will be just uncompressible; they coincide with their shortest gener- 
ation program rather than being random. For the very reason that they are generated by a formal program (the finite sequence itself), they are predictable. It is necessary to have an underlying (generating) physical process in order for a finite sequence or for the production of a single number (the 0 or 1 of a toss of a coin) to be considered as random: unpredictability then, and hence even finite randomness, is once again the result of a friction between Mathematics and the world. In a system of equations or for an evolution function, having no reference to a physical process, but which satisfy the mathematical definition of chaotic determination, randomness is asymptotic, as is Martin-Löf type of randomness (which is well defined at infinity).

The problem is precisely that of the intended meaning of the given mathematical formalism, that is, of the relationship to the process one wants to formalize/modelize. Or, better, in the measurement which enables to pass from the physical process to the mathematical system. When this is an interval, one cannot provide the mathematical model with an exact value, even less than with an integer or with a computable number and, in non-linear dynamics, the input interval is "mixed" and (exponentially) extended through the evolution (over time, generally). This makes the modelling mathematical system obviously computable, but unable to predict the evolution of the modelized physical process. This does not prevent very important qualitative information from making formalization of great interest (this is Poincaré's geometry of dynamical systems, but Hadamard as well should be quoted, for his early work on the geodetic flow on hyperbolic surfaces).

To summarize, it does not make sense to speak of the unpredictability of a mathematical system, even a chaotic one, if it is not in relationship with a (presumed) physical process which it modelizes: it is the latter which will be unpredictable (relatively so); the Mathematics, for their part, are (almost always) computable. There are also systems and processes which are deterministic and predictable, that is, Laplacian ones: in this category we find processes of which the modelization is well expressed by linear (continuous) systems, or by systems of which the relevant data bases are discrete. In both cases, the problem of measurement does not have any important consequence (linearity: the interval is not "mixed") or is not an issue (discreteness: each datum is well separated and accessible, exactly).

In the second case, the difference, I insist, is due to the exact nature of the discrete database, "digit by digit", well separated the ones form the others, without the problem of measurement: its "natural" topology (and the term "natural" has a mathematical sense) is discrete, it isolates each point from the other and enables to access it with exactitude). By physically realizing Turing's discrete state machine, we dared to invent a physical process where measure is exact, in contrast to what happens for all (classical) processes ${ }^{2}$. Moreover, iteration, which is also a form of prediction, is a constitutive principle of Computer Science, daughter of Formal Arithmetics: Herbrand and Gödel's primitive recursion, since 1930-1931, when the first ideas on computability appear, is it-

\footnotetext{
${ }^{2}$ Unfortunately, computer scientists, reverse the names and call exact in reference to physical measure (in a continuum), and approximated the round-off.
} 
eration (in addition to the updating of a register). The portability of software is also a question of iteration, as we said: one wants to be able to run an expensive program identically, so that it may perform adequately and do always exactly the same thing. On the other hand, we summarize and insist, the physical measure (classical and relativistic) is not an integer, but always an interval, which we represent better by means of a Euclidean topology within a continuum: a fluctuation/variation below the interval, therefore an inaccessible one, causes different evolutions in deterministic non-linear or chaotic systems. And in non-linear continuous dynamics, what is most interesting is exactly the role of variation, if possible under the threshold of physical observability, as Turing remarked in his ' 52 paper, a pioneer in this as in inventing the discrete artificial processes. Obviously, these two different mathematical structures construct different ideas of the world; both being very effective in terms of their own objectives, but remaining profoundly different, at least as for proposed causal relations and symmetry breakings (the round-off is a symmetry breaking, at each step of the computation and this is very relevant in computer modelling of non-linear dynamics).

One who does not make the difference and who identifies the physical process and its various mathematical representations, a double pendulum or the toss of a coin, say, which we understand as a dynamics in the continuum, with discrete computational imitation, even enhanced with pretty virtual images of a rolling coin, looses the intelligibility of both processes, and will not manage to do better. How can one indeed "do better" and introduce randomness into a discrete state machine? Concurrent networks and systems enable better imitations than pseudo-random generators: a network of discrete state machines, typically the Web, or a system of concurrent processes (which concur with a same process and are not a priori synchronized by an absolute and common Newtonian clock), that are distributed in space, are indeed immersed in the space time which we understand better by means of continuous Mathematics. The spatio-temporal shifts, even of relativistic type if the network is distributed over the surface of the earth, and multi-tasking, be it local or global, would present phenomena specific to "continuous dynamics". However, if we simulate with network randomness or concurrency the local randomness due to thermal fluctuation in a double pendulum, we will considerably improve the discrete imitation, but will still continue to produce an imitation: we do not take what we consider to be the local cause, inaccessible to measurement, of random variation. But it is already better to import, into discreteness which iterates, the classical or relativist randomness of space-time, which does not iterate.

Let's note, by the way, that Turing, in his 1950 article, says twice that his machine "is Laplacian", because, in such machine, determination implies predictability. "In concrete machines also", he insists. Let's restate it like this: prediction is possible, at least by iteration, even if there are tricks, not inherent to either sequential or concurrent calculus, which can nicely imitate randomness. On the other hand, his 1952 system for morphogenesis is profoundly non-Laplacian and such is its most important property, Turing emphasizes: the dynamics of forms always vary, are deterministic and unpredictable, because are very sensitive to the initial conditions. 
I spoke earlier of microphysics, and I would like to mention that it is now possible to enhance a computer with the randomness of Quantum Mechanics, modifying even more deeply the nature of our digital machines, which are deterministic in the Laplacian sense. Actually, it is possible to improve the computational imitation of classical randomness (dice, the coin) with "intrinsic" forms of randomness thanks to Quantum Physics. We can purchase a box in Geneva which produces 0 s and 1s, according to the up spin and down spin of an electron. In this case, the standard theory says: the probability is intrinsic, because it is the theoretical consequence of the principle of indetermination of Quantum Physics and of quantum measurement, which is always a value of probability. From the standpoint of the analysis of the 0-1 sequence, the probability distribution is analogous. But the difference is radical with regards to both the classical digital computer and to the toss of a coin (the quantum probabilities are "entangled", see [Bailly F., Longo G. 2007]. It is then a question of three different structures of randomness which can at best resemble each other by possible reciprocal imitation, but nothing more ${ }^{3}$.

\section{Calculus, Physics and living phenomena}

I hope that everything we have covered, the dualist and alphabetical nature of these extraordinary machines, the specificity of their causal, Laplacian regime as Turing observed, the difference between computational imitation and physicomathematical modeling, helps to perceive the immense yet singular role of Computer Science for the sciences. When we see human beings moving or cells developing in a virtual context, I hope the reason why they seem a bit strange is clear: the dynamics of the images, even their aesthetics, gives an intuition of it at first glance: they actually iterate, and that is what produces this sensation of something... artificial and unaesthetic. Indeed, you know perfectly well that if you press "restart", they will make exactly the same movements (when has anyone seen a group of extras on a movie set or a group of monocellulars who,

\footnotetext{
${ }^{3}$ In the computer scientist jargon, a formal process is called "non-deterministic" when it is described by a non-functional relation (a non-deterministic Turing Machine, for example: to one input value, there correspond many outputs). We are then simply away from the classical notion of (mathematical) determination, for which a given evolution function or the solution of the intended system of equations, if any, is supposed to be "single-valued". Then and in contrast to the claim by many, in this case randomness and unpredictability are not at stake: the formalism simply does not describe a deterministic process, whether chaotic or not. It is a non-functional formalism, where an input number does not determine an output number, but an entire set of them. Unpredictability and randomness may be called upon if one happens to associate the non-functional relation to, say, the measure of a quantum process: the result then may be considered a possible (random) value amongst probable ones, thus unpredictable, in the interface between Mathematics and physical processes, as usual. We then get into quantum randomness, which we mentioned and which differs from classical randomness as related to deterministic chaotic systems, with their peculiar properties of sensitivity to initial conditions etc.... In this and many other cases, the conceptual confusion is often remarkable in unexperienced people and lead to the vision of a "Computing Nature". The point is that Frege and Hilbert have forbidden to relate the foundation of Mathematics (as Logic or formal systems) to physical space and time, or meaning in them, and Computing originated from Logic, programmatically far away then from natural sciences. Without their courageous step, of which they were largely aware, we would not have these fantastic artifical machines, but we must now be as well aware of the conceptual gap we created by the peculiarities of the arithmetic tool.
} 
if we produce them again in their Petri, will take exactly the same trajectories, make exactly the same movements?). The astute creator of virtual realities, if required to, will imitate physical (and animal) variation by means of variegated effects (economical, classical pseudo-randomness generators or using temporal shifts by multi-tasking or concurrency), but often, the designer will not think of it and the "restart" will be somewhat disappointing. It is not a question here of life or of "will" et similia: I have recently seen some very nice images of lots of balls bumping into each other but... after having pressed the "restart" button, they took the exact same, identical trajectories. Try to make a real swarm of physical balls circulate and bang each other: you will see a different dynamic each time (the programmer immediately improved the imitation in question, by means of network's randomness). One must be cautious, because what I am trying to describe is an evocative problem, an issue for the imagination, one of great scope: it is a play between the representation, the model, and the imitation of dynamics that is at the center of scientific intelligibility and, I would add, of human intelligibility. Without speaking of human movements which are not balls and which are complicated by a series of other causal elements, as always among living phenomena. This, for intelligibility, is a huge problem that has not yet been fully analyzed. Computational simulations cost a lot less than experiments: hence, many physicists renounce to conducting experiments and work only on implementations. The simulation of turbulence, an extreme case of chaos, not only enables to save on wind tunnels, but its iterability is also an asset: the expert's qualitative judgment at a glance may require as many iterations as necessary in order to appreciate the behavior of an airplane's wing or of the cockpit, and the small variations induced give a good appreciation of the dynamic's sensitivity (but this does not enable to analyze an assemblage of wing and cockpit: virtuality in this case is too far removed from the phenomenon which is excessively complex).

The debate in Physics in this regard becomes more profound and with intelligence: theorems of stability or of "shadowing" (the physical or continuous trajectory "follows" the virtual trajectory), in some cases, make explicit that which discrete simulations show us: the analogies and differences with regard to processes which we understand better by means of the analysis of the non-linear continuum.

To summarize, in computational imitation one can have a very original detachment from the world, which is a possible asset if it is well understood. The digital world is an extraordinary invention, as important as the alphabet of which it is a further extension, as I was saying. But we should know how to remain in our knowledge, how to grasp its originality with regard to previous history or the way our knowledge reproposes each time a different perspective on the world: like the alphabet, which did not exist prior to its very audacious invention, the computer is a very original proposition by mankind, it shapes our way of constructing knowledge, it marks it with its own constitutive logic, its own causal regime. We are now able to construct, by means of imitation, absolutely fantastic structures that are, if completely static, true models. However, it must be clear that, each time a dynamic is involved, the imitation can differ completely from the modelization. What is needed is only some element of a non-linear dynamic or a bit of something human (or animal). In physical (and 
biological) dynamics, variation also counts, and very much so; in particular, the variation below the threshold of observability, which rapidly modifies the same observable processes. The computer, which has a single "intrinsic" level of observability, that of digital round-off, which is specific to its discrete data structure, does not know how to capture such a variation, the one which counts the most in sensitive dynamics, and for this reason, it can iterate in an always identical manner.

In the imitation of living phenomena, the problem is particularly serious. If we perform a virtual animation of a living organism, we will right away have the impression that something is wrong, as I was saying, because variability is at the center of life, exactly in the way that identical iteration is at the center of digital computation. What counts in living phenomena, is that a cell is never identical to a mother cell: the orgin of the phylogenetic drift; then, we have Darwinian selection. At the cognitive level, an action is never identical to a preceding action, though it may sometimes bear a close resemblance. The difference in this case is particularly marked, also with regard to the physical notion of variation, because variability includes the individuation of living entities (its "specificity" with regard to the "genericity" of the physical experimental object, see [Bailly F., Longo G. 2006]). Although there does exist in living phenomena a very rigid chemical fragment of phylogenetic memory, DNA, it is only a component of the ontogenetic dynamic: with RNA and non-linear reciprocal interactions, it is at the origin of the proteinic cascades which occur during mitosis, meiosis and embryogenesis, in one of the most complex and least understood dynamics there are. DNA is of course a very important component from the hereditary point of view but, from the cell structure to the epigenetic context, many other factors contribute to ontogenesis; particularly, a multitude of irreproducible and irreversible dynamics, which are at the center of the variability of living phenomena. In this case also, the presumed Democritean alphabet and the notion of program are absolutely insufficient (causally incomplete, see [Longo G., Tendero, P.E. 2007]) for understanding the biological dynamic in which non reversible and uniterable processes contribute in an essential way to the production of ontogenetic and phylogenetic variability, without which there would be no evolution nor life.

I would like to insist here on a later distinction and precision concerning the more general difficulty of making life intelligible using our current mathematical tools. Mathematics is a science of invariants and of the transformations preserving them. We begin with rotations, translations, Euclidean homotheties that preserve symmetries, up to transformation groups and invariants, as in Klein's classifications of various Riemannian geometries (Euclidean, elliptic, hyperbolic). The (mathematical) theory of categories explains this well, by identifying the objects (invariants) and the transformations which preserve them (morphisms, functors, natural transformations). Discrete Mathematics, and hence Computer Science, adds invariance to this by iteration, a sort of symmetry by temporal translation. What can one say when Mathematics, constructed this way, are applied to the analysis of living phenomena? Where can one find as much conceptual and Physical stability? At the phenomenal level, life exists precisely by opposite properties: next to "structural stability", a very weak form of invariance, variability is perhaps the main "invariant", because 
without it there would be no phylogenetic drift, in other words, no evolution. And no ontogenesis, with its variability and non-iteratability of relevant processes. Structural stability does not have the characteristics of mathematical invariance, despite the attempts by René Thom to grasp it using the instruments of Singularity Theory (and the successes in the analysis of the morphogenesis of some organs, in phyllotaxis in particular).

The efforts of some research groups (including mine) consist in identifying the invariants that are specific to living phenomena and are insufficiently described by current physico-mathematical theories. We speak of this in the book we mentioned, where we put the emphasis on the great temporal, even inter-specific invariants, and also on a notion derived from Physics, but which is unsuitable for known physical dynamics, that of the "extended critical situation". Living phenomena, we conjecture and try to express rigorously, would find itself in a singular mathematical situation in the technical sense, usually punctual in Mathematics, but yet extended, in this case, in a non zero measure space, a spatio-temporal interval.

\section{$5 \quad$ But... natural processes compute?}

Let's take once more a step back into history. In the 30s, from the works of Herbrand and Gödel, numerous formal systems for computability enabled to make rigorous the intuitions of the founding fathers of Mathematical Logic (Peano and Hilbert, among others): the deductive certainty of Mathematics lies in its potential mechanization. It was then an issue of associating with formal deduction, so clearly defined by Hilbert and his school, an adequate mathematical notion of effective calculus or of "potentially mechanizable". And this in the domain of systems based on Arithmetics, which Frege and Hilbert had set at the center of the foundational project, and for good reasons at that: the profound crisis which toppled the geometrical certitudes of Euclidean space. Over the course of the following years, Church, Kleene and others proposed other logico-formal systems, which were apt to grasp this originally informal notion of effective deduction. The breakthrough however, the forerunner of Computer Science, occurred in the years 1935-1936: at that time, Turing invented his "abstract" machine, and Turing and Kleene demonstrated the equivalence of various formalisms for effective calculus, all being grounded on integer Arithmetics, of course. But why would the Turing Machine (TM), beyond the demonstrated universality (invariance) of computational systems, have such an important role for the successive developments of Computer Science? Well, some other formalisms would in fact be much better, from several points of view, and more interesting from the mathematical standpoint.

The lambda calculus by Church, for example, possesses "specific theorems" which are very interesting (Church-Rosser, Normalization...) and which rigorously correlate the notion of calculus to that of formal proof, which is the aim of such works (certainty in a proof is the effective computability of it, they said). Conversely, the TM does not possess interesting theorems of its own and if it is used for purposes of demonstration, for complexity analyses for instance, 
one is eager to prove that they are independent from the chosen computational formalism (modulo some "simple" translations). But Turing's system expresses better than any other the nature of the effective computation: it is a writingrewriting of the numbers and of the very rules for the calculation. The Logical Computing Machine, as the author calls it in 1936, writes or deletes 0 or 1 , moves a read/write head one notch to the left or to the right over a ribbon, by changing its internal state at each step, on the basis of a finite set of instructions (here is the afore-mentioned distinction between hardware - ribbon and head and software, the instructions: write delete, move left-right, change state).

Inspired by Gödel, Turing codifies also the instructions with 0s and 1s: on the ribbon, the instructions themselves can be written and modified, as numbers. A machine whose ribbon has instructions and inputs written on it and which is programmed to apply the former to the latter, the Universal Machine, will become the model for modern compilers and operating systems, in short, the model for Computer Science, even current. Hence its mathematical and practical importance: it makes explicit the computation in its elementary and simple components, as transformation of numbers and of programs on numbers, them too being codified by numbers. And this because the calculation is the writing-rewriting of numbers.

Yet, some claim that Nature computes. What sense does it make then to wonder if this table, a waterfall, a strike of lightening, a falling body, a double pendulum, an electric current, a growing tree, a quantum dynamic... any natural process, computes? To make them compute, it is necessary, first of all, to decide where is the input (when the computation begins), where it ends (the output), and then associate numbers to them. In other words, it is necessary to associate these pre-chosen input-output states/instants to numbers by means of physical measurement. Since Riemann-Einstein or Poincaré and Planck, it had been understood that this process, the measurement, has a huge importance for Physics: reference systems and measure are crucial in Relativity; the evolution of a chaotic dynamic can depend on fluctuations or variations below the threshold of possible measurement; quantum indetermination, a key property of measurement, has changed Microphysics. But Frege and Hilbert have forbidden us to think to the guys above when doing foundations of Mathematics, as if this foundation could be detached from that of Physics (see [Bailly F., Longo G. 2006]) and lead us to excellent Mathematics (Logic), to a fantastic digital device and... to the catastrophic philosophy of nature that largely accompanied Logic and Computing or their vulgarization (the brain, the DNA, the Universe. . . are a big Logical Computing Machine; a laplacian one, of $\left.\operatorname{course}^{4}\right)$.

I think that, as first approximation, we should instead assert that

no natural process computes.

And this is due to the key issue, for modern non-laplacian Physics: the issue of measure and access to phenomena. Yet, following a path that went from

\footnotetext{
${ }^{4}$ Fortunately, some have started to reverse the trend and open Logic to the world. By symmetries in rules, for examples, or by ideas inspired from the non-commutative geometry, based on Quantum non-commutative measurement.
} 
the invention of the number, of course rooted in pre-human, animal practices of "small-scale counting", and from the writing of the number and of the alphabet which alone enabled to conceive the numeric codification of meaningless letters ${ }^{5}$, we have achieved the masterpiece of formalising the alphanumeric Cartesian dualism which is the Turing Machine. Then, we were able to transfer such a logico-mathematical invention to physical machines, using artificial processes that are truly original, having the rare quality of evolving by discrete states, a quality obtained with great intelligence by means of valves and transistors, diodes and chips. Therefore, at each moment, the access to the data is exact, measurement is certain (and easy), and does not present the problems of continuous dynamics, as Turing observes with a rare lucidity in his 1950 article (only under the bias and the myths of Artificial Intelligence one could fail to grasp his point, see [Longo G. 2007] where that paper is understood in parallel with the 1952 paper by Turing, [Turing A.M. 1952]), nor does it present the problems associated to quantum processes. In short, to make non Laplacian, dynamic or quantum processes "compute", it is necessary to take measurements, and this is a crucial issue for both theories. The huge problem of modern "Quantum Computing" is precisely that "what is computed is not what is measured". In other words, the evolution of a system, described for instance by Schrdinger equations, occurs in Hilbert spaces with complex values and formal calculations, for example the sums that express quantum superposition, are performed within the field of complex numbers. Measurement, on the other hand, is performed within the field of real numbers, by taking the modules of complex values and by loosing that which constitutes the very structure of entanglement. This is the conceptual barrier which still fails to make the numerical use of quantum superimposition or entanglement phenomena something topical: in other words, we are still theoretically far from obtaining, after the measurement, the numeric (real) results which fully use quantum non-separability (the original contribution of Quantum Computing).

It is obvious that some physical phenomena, Laplacian or linear ones for example, enable an easy and effective association of numbers to process and that it is therefore possible to say that they "compute". In chemistry, say, the processes of molecular interaction may be exhaustively described by "discrete state" systems (atom by atom), to a point of making a great part of theoretical chemistry into a "system of alphabetic rewriting". Nonetheless, in general, the problem of measurement or the production of data from the world, a challenge of modern Physics, is not an issue within the inventive audacity of discrete state, digital, Computer Science. Adressing this problem confers precise meaning to otherwise vague and wild imaginings about computation and nature: in order to associate a physical process to numbers and to an input/output computation, it is necessary to perform a measurement.

And here lies one of the reasons for the lack of success of analogous computations. Created prior to Turing's type of discrete computability, the Differential Analyzer by V. Bush from MIT was, for example and since 1931, a splendid system of analog integration (a little bit in the way that a surface "computes"

\footnotetext{
${ }^{5}$ In the many Chinese war treatises there exists no cryptography, already present with Caesar or in the biblical Kabala; at most, in Chinese, a concept would be evoked instead of another, in order to deceive the enemy who remained uninformed of the ambiguity game.
} 
a curve's integral): it was later on developed by Shannon in 1944 as the GPAC (General Purpose Analog Computer). But, once again, the approximation of the measurement, the low effectiveness of the underlying continuous process, the uncertainty of iterability and portability, all these blocked its developments. And there were probably other reasons too, such as the effectiveness of digital technology (its compressibility and its varied codificability: how can one analogically transfer via a telephone cable the equivalent of 20 megabytes, thus providing digital TV, the Internet and unlimited phone services?), but also, maybe, the conveyance of an arithmetico-linguistic prejudice. Mathematical certainty lies in Arithmetics, all foundationalists will say from Frege and Hilbert onwards; knowledge is in language, will say Frege and the analytical philosophers, especially from the Vienna podium; language, broken down in the alphabet, is codified in Arithmetics (Gödel and Turing). And the virtuous-vicious circle sets in, excluding the rest: Arithmetics Language (Arithmetic) Machine and back.

To return to the alphabet, to think that natural processes compute is like thinking that we produce sequences of letters when we speak. It is a "comic strip" vision of language; western comic strips, that is, because Chinese children certainly think that when speaking, humans produce ideograms which are concepts and sounds, as in their comics. And we do emit a continuous song, decomposed by our very audacious ancestors from Mesopothamia into a musical-alphabetic notation, who linked writing and song together by means of the phoneme. An undertaking with deep historical roots, yet conventional. Try transcribing an animal's cry or song: in the four languages which come to my mind, the dog's bark is transcribed as bau-bau, arf-arf, bu-bu, woof-woof. However, I have observed that dogs do bark the same way in the four countries in question. The transcription of Keshua, an exclusively spoken Andean language, was a difficult and highly controversial undertaking, for being transcribed into Latin letters (and why not into Arabic or Jewish alphabets? a pure contingency of history). Typically Spanish phonemes were forced upon it, while modeling, while forcing it into a stream and transforming the language which had an obviously very original musicality. No, we do not produce letters when speaking, in the same way that natural processes do not produce numbers and do not compute, and the mediation of measurement is a critical node. Grasping this point is essential to making the most of our extraordinary human logico-mathematical and then physical invention, the discrete state arithmetic machine, the electronic-digital calculator. And maybe, begin to think of the next machine: history is not over, with digital computability.

\section{Mnemonic interlude}

After an excess of mathematical evocations, I would like to comfort the reader by brushing, briefly and less formally, the issue of memory. It is a curse that the same word is used in reference to animal (human) memory and to digital databases. The difference is abysmal indeed. What is most important in human (and animal) memory is forgetfulness. Forgetfulness is constitutive of invariance and, therefore, of conceptual abstraction, because in this way, we can forget the details, that which are "unimportant". Let me explain. We do not remem- 
ber an image, an event, pixel by pixel, exactly. Our view or understanding is intentional, from the onset, that is, that there is a "goal", an objective to our comprehension or emotions, an intent in our reading of the world which is always active, and which selects that which must be preserved by memory, that which is of interest. Our perception of the world is always a hermeneutic. Memory, moreover, evokes and causes one to relive events by reconstructing, each time at least a little bit differently, the image, the event, by interpolating, by revisiting meaning, by emphasizing one trait instead of another. Never will memory reproduce experience exactly, pixel by pixel. And so memory contributes to abstracting "that which counts", it proposes and constitutes invariants, that is, traits, gestures, "Gestalts" and then relatively stable concepts, which language and writing contribute to make common and to later stabilize, to make them relatively independent from transformations in the ecosystem.

And so it reconstructs while forgetting the relatively insignificant elements, those which are insignificant with regard to our goals, by jettisoning that which is useless. This way, we can recognize a school mate thirty years later thanks to his smile, which is a movement, or thanks to a certain tilt of the head, or a fold which forms under his eyes when he speaks. These are dynamics which are all important in our old affective rapports. Pixel by pixel, this face has nothing in common with the one from 30 years earlier: movements, selected by us as invariants and intentionally meaningful, are all that remain. But that is enough for us, and it is precisely what counts: to have forgotten the exact face, in this case, is fundamental in order to recognize the new, because the old one and its details no longer exist. And all this is the opposite of digital memory, which must be exact: what a disaster it would be if, when opening a file a year later, a comma was to be in a different place. What a disaster it would be if a Web page, opned a second time, were to be scrambled due to a memory or communication failure. In computer science, everything is done in order for databases (and communication) to be exact, pixel by pixel. The Web (Internet), this extraordinary "data base" for humanity, potentially available to all, must be exact: there lies its strength. Of course, even the Web is dynamic and "forgetful": sites will appear and disappear, and they are modified. But this will be due to human intervention: the network of machines must have, in itself, an exact and perfect memory. This is the opposite of intentional, selective and constitutive dynamic of meaning and invariance, in the variability, in the active forgetfulness which is animal memory, in which the forgetting of irrelevant details contributes to the construction of the relevant invariant, the very intelligibility of the world. The extraordinary interest of the Web resides precisely in its role as complement (for its originality as a human invention, in my view one as important as the invention of the printing press) to the forgetfulness and the dynamic of our animal memory a memory which language, writing and the printing have already considerably enhanced for mankind, by contributing to its stabilization. What a mistake to believe that the relevance of digital computing was to be the artificial copy or replacement of human intelligence: it did much more, it enriched it in a revolutionary way. 


\section{Conclusion: A question of principles}

In this short presentation we have attempted to highlight some "principles" or foundational elements which govern great mathematical options for the intelligibility of natural phenomena. If we consider the objective of this informal remarks, being mainly Computer-Science oriented, we have not addressed with sufficient detail the direct and highly fecund relationship between Mathematics and Physics, even if it was always in the background. Specifically, we have only brushed upon the resemblance of the great principles of conceptual construction, between Physics and Mathematics, which justify the "very reasonable" effectiveness of Mathematics in Physics (there are, as to say, "co-constituted", as stressed in [Bailly F., Longo G. 2006], in contrast to the arithmetized foundation, Frege-Hilbert style). And only will such an analysis enable to better grasp the limits of mathematical or digital modelization in Biology, even to move forward, maybe with new ideas (and conceptual structures). The identification of order or symmetry principles, in Mathematics, or the highlighting of the foundational role, in Physics, of the geodesic principle, as we did in [Bailly F., Longo G. 2006], must be developed in order to grasp "that which underlies" and which unifies or distinguishes whole branches of knowledge, the choice of methods and of instruments, explicit and implicit, the constitution of their meaning or the "origin", in an often more conceptual than historic sense, but also in a historical sense. And this in order to question these very principles, if necessary and if that enables to make other fragments of the world intelligible. Indeed, on the one hand, understanding that common construction principles, from Euclid to Riemann and to Connes (the major name in contemporary Geometry of Quantum Mechanics), on the basis of the access to and measurement of space (from Riemann's rigid body to Heisenberg's non-commutative matricial algebra, to which Connes refers), by founding geometric organization, reinforces the sense of each corresponding theory, all the while grasping the radical changes of perspective provided by each of these approaches. Likewise, the fact of highlighting that the geodesic principle may make intelligible a scientific span going from Copernic and Kepler to Schrödinger's equations (derivable from Hamiltonian optimality, as are Newton's equation, in suitable abstract spaces) enables to grasp, in a single glance, the strength of the theoretical proposition in modern Physics, in its successive developments. On the other hand, the "foundational" operation, which counts for us too, consists in a critical "reflection" upon the principles of each science, of "taking a step to the side", of looking at them from a distance, even in order to put them into question, particularly when turning to other scientific fields.

This is what we do when observing, in the book with Bailly, how the phylogenetic (and, in part, ontogenetic) "trajectories" of living matter must no longer be understood as "specific" (geodesics), but rather as "generic" ("possibilities" of evolution), whereas it is rather the living individual who is "specific". In other words, in Physics, the (experimental) object is generic (a body, a photon... can be replaced by any other, in theory and in experiments) and follows specific "trajectories" (critical geodesics), in opposition to Biology. It is a duality with Physics which enables to appreciate the necessity of a theory specific to living phenomena and which enriches the underlying physical principles - which also participate to the intelligibility of living phenomena. It is the foundational anal- 
ysis conducted in the book which should enable to highlight the strength and the limitations of the physico-mathematical and computer science framework, its non absolute character, and the boundaries of its universality. A framework which therefore needs to be completely rethought outside of its historical fields of construction: the very fruitful relationship between Physics and Mathematics.

The aim of a foundational analysis today is certainly not that of the founding fathers who rightly sought certitudes during a period of great foundational crises, particularly that of the crumbling of absolute Euclidean time-space, a goal which was highly justifiable 100 years ago and which was already put into question by a few, including the second Wittgenstein (some still existing logician philosophers reveal rather psychotic traits, in their quest for "unshakable certainties"). Today's aim is rather one of practicing an "ethic" of knowledge, in order to move forward: the duty of each researcher of making explicit the great organizing principles of his or her knowledge, of viewing them with a critical eye, in order to do better, especially by turning towards other scientific fields, where they may be insufficient for understanding or may be put into question, even radically, as happened in both Relativity and Quantum Mechanics. This is the sense of the dynamic universality specific to scientific knowledge, which is quite different from any form of absoluteness.

Particularly, it is important to be cautious with regard to these extraordinary images provided by the discrete state machine: they are rich of knowledge, but propose an understanding of the world which is deeply rooted in the principles of alphabetic representation/reduction and even more so on atomism, dualism and iterability, which are insufficient today for understanding physical processes, and even less so for understanding those specific to living phenomena. Contemporary science, however, with its technical depth and strength, could not exist without the digital simulation and, in general, without the contribution of Computer Science: for this reason, it is necessary to develop a scientific analysis of what it says, precisely, by putting aside, in the same way that Vaucanson's mechanical puppets were quickly forgotten, the myths of a computational Universe, of digital calculating brains, of genetic "programs" and other projections of latest available technologies upon phenomena, an increasingly ridiculous reading of the world, with its iteration over the centuries.

\section{References}

[Anandan J. 2002] Anandan J. "Causality, Symmetries and Quantum Mechanics" Foundations of Physics Letters, vol.15, no. 5, 415 438, October, 2002.

[Alligood K., Sauer T., Yorke J. 2000] Alligood K., Sauer T., Yorke J., Chaos: an introduction to Dynamical Systems, Springer, New York, 2000.

[Bailly F., Longo G. 2006] Bailly F., Longo G., Mathématiques et sciences de la nature. La singularité physique du vivant, Hermann, Paris, 2006 (Introduction in English and in French, downloadable, as Longo's papers below). 
[Bailly F., Longo G. 2007] Bailly F., Longo G., "Randomness and Determination in the interplay between the Continuum and the Discrete". MSCS, to appear, 2007.

[Bell J.S. 1964] Bell J.S., "On the Einstein-Podolsky-Rosen Paradox", Physics 1, p.195, 1964.

[Bitbol M. 2000] Bitbol M. L'aveuglante proximité du rel, Flammarion 2000.

[Bitbol M. 2000] Bitbol M., Physique et philosophie de l'esprit, Flammarion, 2000 .

[Bohm D. 1951] Bohm D., "The Paradox of Einstein, Rosen and Podolsky", Quantum Th. p.611, 1951.

[Bohm D. 1987] Bohm D., La plénitude de l'univers, Paris, Le Rocher, 1987.

[Cappuccio M. 2003] Cappuccio M.. "Traces of computational mind: From wax tablets to Turing machine", Géométrie et cognition, (Longo ed.), Editions rue d'Ulm, Tome 124, s.5, 43- 60, 2003.

[Devaney R. L. 1989] Devaney R. L. An Introduction to chaotic dynamical systems, Addison-Wesley, 1989.

[Herrenschmidt C. et al. 1996] Herrenschmidt C. et al. (eds), L'Orient et nous, Albin-Michel, 1996.

[Hoyrup M., Kolcak A., Longo G. 2007] Hoyrup M., Kolcak A., Longo G. "Computability and the Morphological Complexity of some dynamics on Continuous Domains". Invited survey TCS, to appear, 2007.

[Laskar J. 1994] Laskar J. "Large scale chaos in the Solar System", Astron. Astrophys., 287, L9 L12, 1994.

[Lighthill J. 1986] Lighthill J. "The recent recognized failure of predictability in Newtonian dynamics" Proc. R. Soc. Lond. A 407, 35-50, 1986.

[Longo G. 2007] Longo G. "Laplace, Turing and the "imitation game" impossible geometry: randomness, determinism and programs in Turing's test". In Epstein, R., Roberts, G., \& Beber, G. (Eds.). The Turing Test Sourcebook. Dordrecht, The Netherlands: Kluwer, 2007.

[Longo G., Tendero, P.E. 2007] Longo, G. et Tendero, P.E. "The causal incompleteness of Programming Theory in Molecular Biology", to appear. A french version will appear in Evolution des concepts fondateurs de la biologie du XXIe siècle, DeBoeck, Paris, 2007.

[Mugur-Schächter M. 2005] Mugur-Schächter M., "Probalilités, rélativizations descriptionnelles et complexité", preprint, Paris, 2005.

[Rojas C. 2007] Rojas C. Computability and Information in models of Randomness and Chaos. MSCS, to appear 2007.

[Sini C. 1994] Sini C. Filosofia e scrittura, Roma-Bari, Laterza, 1994. 
[Turing A.M. 1950] Turing A.M., "Computing Machines and Intelligence", Mind, vol. LIX, n236, p. 433-460, 1950.

[Turing A.M. 1952] Turing A.M., "The Chemical Basis of Morphogenesis", Philo. Trans. Royal Soc., vol. B237, p. 37-72, 1952.

[Zurek W.H. 1991] Zurek W.H. "Decoherence and the Transition from the Quantum to the Classical", Physics Today, p.36 October 1991. 